\title{
Editorial \\ Surface Plasmon Resonance (SPR) to Magneto-Optic SPR
}

\author{
Conrad Rizal ${ }^{1, *,+, \neq(\mathbb{D}, \text { Vladimir Belotelov } 2,3, *, \ddagger}$, Daria Ignatyeva ${ }^{2,3} \mathbb{D}^{\circ}$, Anatoly K. Zvezdin ${ }^{2}$ and \\ Simone Pisana ${ }^{1}$ (I) \\ 1 Electrical Engineering and Computer Science, York University, Keele Campus, Toronto, ON M3J 1P3, Canada; \\ simone.pisana@lassonde.yorku.ca \\ 2 Lomonosov Moscow State University, Leninslie Gory, 1, bld.2, Moscow 119992, Russia; \\ daria.ignatyeva@gmail.com (D.I.); zvezdin@gmail.com (A.K.Z.) \\ 3 Russian Quantum Center, Business-center "Ural", Skolkovskoe shosse 45, Moscow 121353, Russia \\ * Correspondence: crizal@yorku.ca (C.R.); v.i.belotelov@ya.ru (V.B.); Tel.: +1(647)570-4039 (C.R.); \\ +7(926)-733-7189 (V.B.) \\ + Current address: Nanophotonics Laboratory, GEM Systems Inc., Markham, ON L3R 5H6, Canada. \\ $\ddagger$ These authors contributed equally to this work.
}

Received: 19 May 2019; Accepted: 22 May 2019; Published: 27 May 2019

check for updates

\begin{abstract}
In this editorial, a brief background of the surface plasmon resonance (SPR) principle is discussed, followed by several aspects of magneto-optic SPR (MOSPR) and sensing schemes from the viewpoint of fundamental studies and potential technological applications. New sensitivity metrics are introduced that would allow researchers to compare the performance of SPR and MOSPR-based sensors. Merits of MOSPR over SPR based sensors and challenges faced by MOSPR sensors in terms of their practical use and portability are also considered. The editorial ends with potential new configurations and future prospects. This work is considered highly significant to device engineers, graduate and undergraduate students, and researchers of all levels involved in developing new classes of bio-devices for sensing, imaging, environmental monitoring, toxic gas detection, and surveying applications to name a few.
\end{abstract}

Keywords: plasmonics; magnetoplasmonics; magneto-optics; SPR; MOSPR; sensing

\section{Background}

The interaction of optical radiation with surface plasmons (coherent delocalized electron oscillations at a metal-dielectric interface) in the absence and presence of a magnetic field $(\mathrm{H})$ resulted in the discoveries of surface plasmon resonance (SPR) and magneto-optic SPR (MOSPR), respectively. The discovery of SPR phenomena dates to the early 20th century, between the years 1902 and 1912, first experimentally demonstrated by R.M. Wood [1], and later theoretically described by Lord Rayleigh in 1907 [2]. However, it was not until 1968 that a convenient approach for observing the SPR phenomenon was introduced by Kretschmann [3] and Otto [4]. Since then, several other researchers and companies continued investigating the phenomena for possible use in the medical field. However, it was only in 1990 that BIACORE commercially developed (first batch of 35 units) an SPR-based device for practical biosensing purposes [5]. Since then SPR has remained a primary choice for biosensing and drug discovery [6].

Surface plasmon polaritons (SPPs) are collective oscillations of the free-electron gas at the boundary/surface of metal and dielectric layers. The wave vector of these waves is given by $k_{s p p}=$ 
$\left(k_{0} \times n_{m} \times n_{d}\right) /\left(n_{m}+n_{d}\right)^{1 / 2}$, where $k_{0}$ is the wave vector in free space and $n_{m}$ and $n_{d}$ are the metal and dielectric refractive indices, respectively. The wave vector therefore depends on the optical properties of the metal and dielectric media, the excitation wavelength (through the dispersion of the optical properties), and excitation conditions. A change in the optical properties of the dielectric media in the region where the waves extend will therefore affect the SPP wave vector and the coupling conditions (i.e., angle, wavelength, intensity, and phase). This feature serves as the foundation for development of the SPR sensors. However, the challenge for conventional SPR-based sensors is to extend their detection limit to lower concentrations and smaller molecules. Magneto-optic modulation techniques can be adopted to improve the performance and enhance the sensitivity.

SPR sensors are based on the interaction of optical radiation with either localized (for structures smaller than the wavelength of incident light) or propagating (for structures larger than the wavelength of incident light) surface plasmons at the interface of two materials having opposite refractive indices. The principle of operation of SPR sensors is based on the detection of the shift in the optical angle of incidence leading to resonance, or intensity changes in the reflected optical signal. These changes are directly proportional to the physical properties of the dielectric media being probed. This medium can be a gas (air, Helium, etc.) or fluid, in particular bio-samples (water, urine, serum or protein, etc.). Sensors based on the SPR principle have been continuously developed and are still active in the market. However, early stage disease detection has remained a very challenging task, and further improvement in detection limits will enable more efficient drug discovery by working with lower-concentration solutions. Currently, SPR sensors have reached their detection limit [7]. For details on plasmonics and SPR, and technical reviews, the readers are referred to [8-13].

The effect of magnetic fields on the optical properties of materials, known as magneto-optic effects, have been observed since the mid-19th century, first studied by Michael Faraday in 1845, well before the discovery of the SPR effect in the metal/dielectric interface. He experimentally demonstrated that an external magnetic field has a direct effect on the polarization of the transmitted optical radiation passed through a dielectric medium. This effect is known as Faraday rotation. In 1877, John Kerr demonstrated a related magneto-optic effect, the rotation of the polarization of light reflected from a magnetic material, known as the magneto-optic Kerr effect. In 1985, the Kerr effect was exploited commercially through a data recording industry. For more details, interested readers are referred to the book by Zvezdin et al. [14].

MOSPR phenomena are relatively newly explored in the field of biosensing and are still in the research and development stage. Lately, their rapid development have led to new physics and the potential for several new applications. The sensors are based on the interaction of magnetic fields with the optical properties of magnetic materials incorporated in the SPR structure. The resulting magneto-optical activity leads to sensitivity enhancement and is also used to modulate the resonance conditions [14]. Although the effect of MOSPR on improving the performance of biosensors has been reported in the literature [15-23], no relevant clinical or commercial application has been realized.

It is important to note that the principle of MOSPR sensing is different from the principle of SPR sensing. Unlike the angular or intensity change measured by SPR sensors in the presence of a target molecule, MOSPR sensors record the derivative of the angular spectra as the magnetic field is varied. This, in principle, allows the optical response to be amplified by hundreds of times to achieve higher sensitivity. Recent studies have shown that MOSPR sensors can indeed outperform SPR sensors in terms of sensitivity and detection limit [24]. The improved sensitivity of the MOSPR sensors would allow for applications beyond biosensing, such as imaging, surveying, and environmental monitoring.

Materials of interest for the development of MOSPR sensors include ferromagnetic layers, multilayers, nanoparticles, alloys, and compositionally graded alloys for the magnetic layer. On the sensor surface, extremely thin dielectric layers have been shown to improve sensitivity and can be used as protective layer. 
In particular, graphene layers have been shown to be effective in this regard, and their carbon surface is also advantageous for the surface functionalization necessary to achieve selectivity [25].

\section{Sensitivity Metrics}

The sensitivity of SPR and MOSPR biosensors is a critical parameter for their benchmarking. However, no unified sensitivity metric is used in the literature to directly compare the performance of these sensors. The problem is that many of the published works are focused on either SPR sensors or MOSPR sensors alone. Moreover, the sensitivity used by device manufacturers are often very specific to a target application. With an aim to provide a guideline for users of SPR or MOSPR biosensors, we present sensitivity metrics as follows, introduced in [26].

Figure 1a shows surface plasmon resonance (SPR) (reflectivity vs. incident angle), and Figure 1b shows magneto-optic SPR (MOSPR) (change in reflectivity due to the presence of a saturating magnetic field $\mathrm{H}$ vs. incident angle) curves for two different media, denoted as A and B for simplicity. According to [26], the SPR sensitivity can be defined as:

$$
\left.S_{S P R}=\frac{\left[R_{p(A)}-R_{p(B)}\right]}{R_{p(A) m}} \times 100\right) / \Delta n[\% / R I U],
$$

and the MOSPR sensitivity is defined as:

$$
\left.S_{M O S P R}=\frac{\left[\left(\Delta R_{p(A)}-\Delta R_{p(B)}\right)\right]}{\Delta R_{p(A) m}} \times 100\right) / \Delta n[\% / R I U],
$$

where, $R_{p(A) m}$ is the magnitude of reflected intensity at an incident angle $\theta_{m}$. This $\theta_{m}$ is the angle at which the first derivative $\left[\left(\delta R_{p(\theta)}\right) / \delta \theta\right]$ is maximized, $\Delta R_{p(A)}$ and $\Delta R_{p(B)}$ are the changes in reflectivity due to the modulating $\mathrm{H}$ field for medium $\mathrm{A}$ and $\mathrm{B}$, respectively, at $\theta_{m}$. The difference $\Delta R_{p(A)}-\Delta R_{p(B)}$ is normalized by $\Delta R_{p(A) m}$, where $\Delta R_{p(A) m}$ is the maximal for the first derivative $\left.\delta\left(\Delta R_{p(A}\right) \theta\right) / \Delta \theta_{(A)}$. The $\Delta n$ in (1) and (2) is the difference in refractive indices between media $A$ and $B$, and RIU denotes refractive-index units.

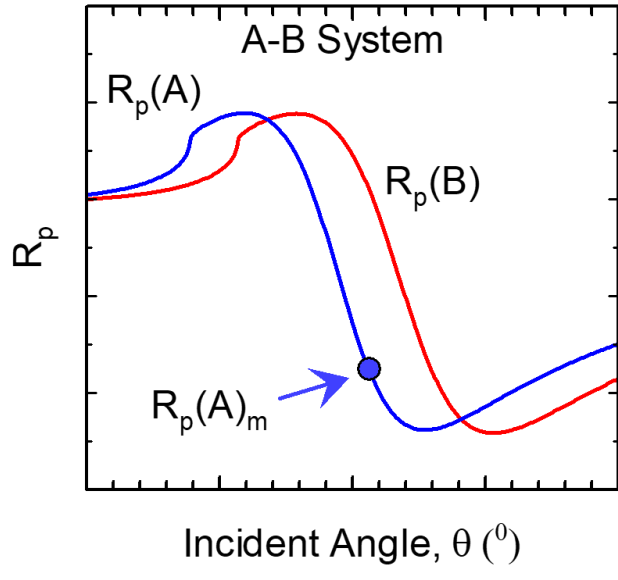

(a)

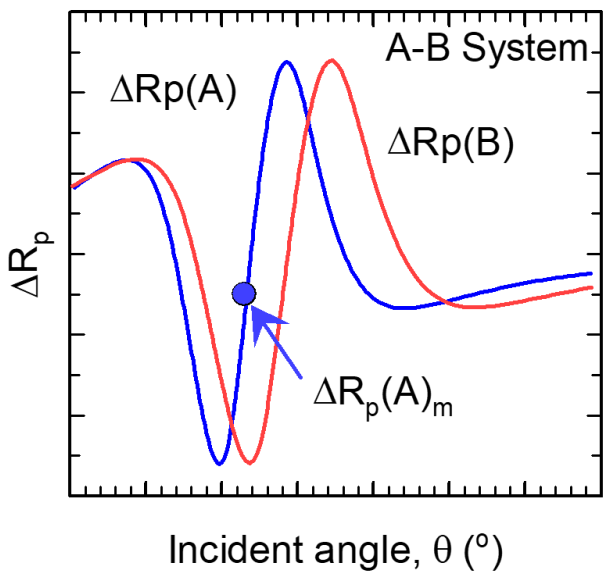

(b)

Figure 1. Comparison of the SPR and MOSPR sensitivities for two systems denoted by A and B. 
These sensitivity metrics eliminate diverging sensor response since both $R_{p(A) m}$ and $\Delta n$ in the SPR case and $\Delta R_{p(A) m}$ and $\Delta n$ in the MOSPR case are never zero at $\theta_{m}$, near the angle $\theta_{S P R}$, as $\theta_{m}$ is chosen where the slope of the curve is maximized. The sensitivity metrics in (1) and (2) can be used to compare the performance of SPR and MOSPR sensors directly.

The quality factor of the sensing structure is crucially important both for SPR and MOSPR sensing, since the steeper the resonance is, the higher response is observed for the same variation of the analyte. In this sense, MO structures using ferromagnetic dielectrics, for example, bismuth-substituted [27] or cerium-substituted [28] garnets, are preferable as they are transparent and do not introduce additional losses, in contrast to ferromagnetic metals such as Co and Ni. On the other hand, utilization of the photonic-crystal structures could also allow for the increase of SPR [29] and, consequently, the MOSPR quality factor [30]. The consequential increase of the sensitivity of photonic-crystal MOSPR sensor was demonstrated [27,31].

\section{Potential Applications}

One area where magneto-optics has been extensively studied is the localized magneto-optic SPR (LMO-SPR) functionality using magnetoplasmonics (magnetic-nonmagnetic) core-shell nano-particles, nano-rods, nano-antenna or nano-wires of dielectric and plasmonic materials [32]. These magnetoplasmonic structures have been considered for potential applications in drug delivery, drug detection, and biosensing, among many others. In addition to the intensity change, plasmon-induced phase modification of the reflected light is also employed as a means of sensing. These same materials can also be used as sensors by analyzing the phase of the transmitted optical radiation as well. Similarly to what was described earlier, the significance of these magnetoplasmonic core-shell structures over the plasmonic core-shell structure is that the tunability of the MOSPR property can be achieved using the magnetic activity. Despite the optical loss induced by the ferromagnetic material, the applied magnetic field has substantial effects on the MO characteristics to yield an overall sensitivity gain.

Another method to stimulate LMO-SPRs involves patterning periodic arrays of nanoholes in magneto-optic-plasmonic multilayers [33]. These new type of nanostructures can find applications in biosensing and photovoltaics [33,34], as welll as in developing improved amplifiers and lasers [35].

Since the discovery of giant magnetoresistance (GMR) in metallic alloys and multilayers, GMR sensors have found many applications in the magnetic data storage industry $[36,37]$. These same materials have also found important applications as biosensors [38,39]. Likewise, metamaterials are another class of materials that are considered important for magnetoplasmonic studies [40]. Magneto-optic functionality can be incorporated in metamaterials to tune the scattering emission or make it vanish entirely. For example, the work presented in [41] can be extended to biosensing and bioimaging applications where the reflection of light through the sensor can be controlled through the addition of magnetic metal and varying the thickness or size of the metamaterial, incident angle, or direction and magnitude of the applied magnetic field.

Optimization of SPR/MOSPR configurations can be performed through simulation based on the Fresnel reflection formalism. Tools such as the transfer-matrix method have been employed to investigate various device parameters necessary to excite SPR and induce magneto-optic enhancement. For a description of theory and modeling of SPR and MOSPR effects in heterostructures, we encourage interested readers to see the following references [42,43]. The simulation results have demonstrated that the inclusion of refractory and ferromagnetic materials into a photonic crystal configuration yields much higher sensitivity and detection level. 


\section{Summary}

The performance of MOSPR sensors have warranted their use in future sensing applications in both the gaseous and liquid media. Furthermore, MOSPR sensing can find applications in determining the physical properties of materials and biosamples. Since the proof of concept has already been developed and the sensing functionality experimentally demonstrated, MOSPR sensors are poised to make considerable impact in the field of biosensing in the near future.

Author Contributions: C.R. wrote the editorial and all authors read, discussed, revised, and approved it.

Funding: The work was partially supported by MITACS Canada (Grant \# 4086), GEM Systems Inc., and York University and by Russian Foundation for Basic Research (Grants \# 18-52-80038 and 18-52-45021).

Conflicts of Interest: The authors declare no conflict of interest.

\section{References}

1. Wood, R.W. On a remarkable case of uneven distribution of light in a diffraction grating spectrum. Lond. Edinburgh Dublin Philosoph. Mag. J. Sci. 1902, 4, 396-402. [CrossRef]

2. Rayleigh, H.L. On the dynamical theory of gratings. Proc. R. Soc. Lond. Ser. A 1907, 79, 399-416. [CrossRef]

3. Kretschmann, E.; Raether, H. Radiative decay of non radiative surface plasmons excited by light. Zeitschrift für Naturforschung A 1968, 23, 2135-2136. [CrossRef]

4. Otto, A. Excitation of nonradiative surface plasma waves in silver by the method of frustrated total reflection. Zeitschrift für Physik A Hadrons and nuclei 1968, 216, 398-410. [CrossRef]

5. Säfsten, P.; Klakamp, S.L.; Drake, A.W.; Karlsson, R.; Myszka, D.G. Screening antibody-antigen interactions in parallel using Biacore A100. Anal. Biochem. 2006, 353, 181-190. [CrossRef] [PubMed]

6. Owen, V. Real-time optical immunosensors-A commercial reality. Biosensor. Bioelectron. 1997, 12, 1-2. [CrossRef]

7. Piliarik, M.; Homola, J. Surface plasmon resonance (SPR) sensors: approaching their limits? Opt. Exp. 2009, 17, 6505-16517. [CrossRef]

8. Stockman, M.I. Nanoplasmonics: past, present, and glimpse into future. Opt. Exp. 2011, 19, 22029-22106. [CrossRef]

9. Atwater, H.A.; Polman, A. Plasmonics for improved photovoltaic devices. Nat. Mater. 2010, 3, 205. [CrossRef]

10. Brolo, A.G. Plasmonics for future biosensors. Nat. Photon. 2012, 6, 709-713. [CrossRef]

11. Maier, S.A. Plasmonics: Fundamentals and Applications; Springer Science \& Business Media: Berlin, Germany, 2007.

12. Monteiro, J.P.; de Oliveira, J.H.; Radovanovic, E.; Brolo, A.G.; Girotto, E.M. Microfluidic plasmonic biosensor for breast cancer antigen detection. Plasmonics 2016, 11, 45-51. [CrossRef]

13. Mayer, K.M.; Hafner, J.H. Localized surface plasmon resonance sensors. Chem. Rev. 2011, 111, 3828-3857. [CrossRef]

14. Zvezdin, A.K.; Kotov, V.A. Modern Magnetooptics and Magnetooptical Materials; Coey, M., Ed.; CRC Press: New York, NY, USA, 1997.

15. González-Díaz, J.; Sepúlveda, B.; García-Martín, A.; Armelles, G. Cobalt dependence of the magneto-optical response in magnetoplasmonic nanodisks, Appl. Phys. Lett. 2010, 97, 043114.

16. Temnov, V.V. Ultrafast acousto-magneto-plasmonics Nat. Photon. 2012, 11, 728-736. [CrossRef]

17. Belotelov, V.I.; Akimov, I.A.; Pohl, M.; Kotov, V.A.; Kasture, S.; Vengurlekar, A.S.; Gopal, A. V.; Yakovlev, D.R.; Zvezdin, A.K.; Bayer, M. Enhanced magneto-optical effects in magnetoplasmonic crystals. Nat. Nanotechnol. 2011, 6, 370. [CrossRef]

18. Chin, J.Y.; Steinle, T.; Wehlus, T.; Dregely, D.; Weiss, T.; Belotelov, V.I.; Stritzker, B.; Giessen, H. Nonreciprocal plasmonics enables giant enhancement of thin-film Faraday rotation. Nat. Commun. 2013, 4, 1599. [CrossRef]

19. Bonanni, V.; Bonetti, S.; Pakizeh, T.; Pirzadeh, Z.; Chen, J.; Noguacs, J.; Vavassori, P.; Hillenbrand, R.; Akerman, J.; Dmitriev, A. Designer magnetoplasmonics with nickel nanoferromagnets. Nano Lett. 2011, 11, 5333-5338. [CrossRef] 
20. Maksymov, I.S. Magneto-plasmonics and resonant interaction of light with dynamic magnetisation in metallic and all-magneto-dielectric nanostructures. Nanomaterials 2015, 5, 577-613. [CrossRef]

21. Pellegrini, G.; Mattei, G. High-performance magneto-optic surface plasmon resonance sensor design-An optimization approach. Plasmonics 2014, 9, 1457-1462. [CrossRef]

22. Regatos, D.; Sepúlveda, B.; Fariña, D.; Carrascosa, L.G.; Lechuga, L.M. Suitable combination of noble/ferromagnetic metal multilayers for enhanced magneto-plasmonic biosensing. Opt. Expr. 2011, 19, 8336-8346. [CrossRef]

23. Wang, L.; Clavero, C.; Huba, Z.; Carroll, K.J.; Carpenter, E.E.; Gu, D.; Lukaszew, R.A. Plasmonics and enhanced magneto-optics in core-shell Co-Ag nanoparticles. Nano Lett. 2011, 11, 1237-1240. [CrossRef]

24. Rizal, C.; Niraula, B.; Lee, H.H.W. Biomagnetoplasmonics, emerging biomedical technologies and beyond. J. Nanomed. Res. 2016, 3, 00059-00065. [CrossRef]

25. Szunerits, S.; Maalouli, N.; Wijaya, E.; Vilcot, J.-P.; Boukherroub, R. Recent advances in the development of graphene-based surface plasmon resonance (SPR) interfaces. Anal. Bioanal. Chem. 2013, 405, 1435. [CrossRef]

26. Rizal, C.; Pisana, S.; Hrvoic, I. Improved magneto-optic surface plasmon resonance biosensors. Photonics 2018, 5, 116. [CrossRef]

27. Ignatyeva, D.O.; Kapralov, P.O.; Knyazev, G.A.; Sekatskii, S.K.; Dietler, G.; Nur-E-Alam, M.; Vasiliev, M.; Alameh, K.; Belotelov, V.I. High-Q surface modes in photonic crystal/iron garnet film heterostructures for sensor applications. JETP Lett. 2016, 104, 679. [CrossRef]

28. Qin, J.; Zhang, Y.; Liang, X.; Liu, C.; Wang, C.; Kang, T.; Lu, H.; Zhang, L.; Zhou, P.; Wang, X. Ultrahigh Figure-of-Merit in Metal-Insulator-Metal Magnetoplasmonic Sensors Using Low Loss Magneto-optical Oxide Thin Films. ACS Photon. 2017, 4, 1403. [CrossRef]

29. Konopsky, V.; Karakouz, T.; Alieva, E.V.; Vicario, C.; Sekatskii, S.; Dietler, G. Photonic crystal biosensor based on optical surface waves. Sensors 2013, 13, 2566. [CrossRef]

30. Ignatyeva, D.O.; Knyazev, G.A.; Kapralov, P.O.; Dietler, G.; Sekatskii, S.K.; Belotelov, V.I. Magneto-optical plasmonic heterostructure with ultranarrow resonance for sensing applications. Sci. Rep. 2016, 6, 28077. [CrossRef]

31. Otipka, P.; Vlceka, J.; Lesnak, M.; Sobotac, J. Design of MO-SPR sensor element with photonic crystal. Photon. Nanostruct. Fundam. Appl. 2018, 31, 77. [CrossRef]

32. Tomitaka, A.; Arami, H.; Raymond, A.; Yndart, A.; Kaushik, A.; Jayant, R.D.; Takemura, Y.; Cai, Y.; Toborek, M.; Nair, M. Development of magneto-plasmonic nanoparticles for multimodal image-guided therapy to the brain. Nanoscale 2017, 9, 764-773. [CrossRef]

33. Rizal, C.; Moa, B.; Brolo, A.G. Recent progress in ferromagnetic multilayer-based magnetoplasmonic devices for potential biomedical applications. Can. Med. Biol. Soc. Ser. 2014, 4, 1-4.

34. Menezes, J.W.; Ferreira, J.; Santos, M.J.L.; Cescato, L.; Brolo, A.G. Large area fabrication of periodic arrays of nanoholes in metal films and their application in biosensing and plasmonic enhanced photovoltaics. Adv. Funct. Mater. 2010, 20, 3918-3924. [CrossRef]

35. Berini, P.; de Leon, I. Surface plasmon-polariton amplifiers and lasers. Nat. Photon. 2012, 6, 16. [CrossRef]

36. Osterfeld, S.J.; Yu, H.; Gaster, R.S.; Caramuta, S.; Xu, L.; Han, S.J.; Hall, D.A.; Wilson, R.J.; Sun, S.; White, R.L.; et al. Multiplex protein assays based on real-time magnetic nanotag sensing. PNAS 2008, 105, 20637-20640. [CrossRef]

37. Ding, Y.; Judy, J.H.; Wang, J.P. Magneto-resistive read sensor with perpendicular magnetic anisotropy. IEEE Trans. Magnet. 2005, 41, 707-712. [CrossRef]

38. Rizal. C. Microstructure, magnetism, surface plasmon resonance, and magneto-optic surface plasmon resonance biosensors. IEEE Trans. Magnet. 2018, 55, 530030.

39. Rizal, C.; Pisana, S.; Hrvoic, I.; Fullerton, E. Microstructure and magneto-optical surface plasmon resonance of Co/Au multilayers. J. Phys. Commun. 2018, 2, 055010. [CrossRef]

40. Rizal, C.; Moa, B.; Niraula, B.B. Ferromagnetic multilayers: Magnetoresistance, magnetic anisotropy, and beyond. Magnetochemistry 2016, 2, 22. [CrossRef]

41. Lu, D.; Kan, J.J.; Fullerton, E.E.; Liu, Z. Enhancing spontaneous emission rates of molecules using nanopatterned multilayer hyperbolic metamaterials. Nat. Nanotechnol. 2014, 9, 48-53. [CrossRef] 
42. Visnovsky, S.; Nyvlt, M.; Prosser, V.; Ferr, J.; Penissard, G.; Renard, D.; Sczigel, G. Magneto-optical effects in $\mathrm{Au} / \mathrm{Co} / \mathrm{Au}$ ultrathin film sandwiches. J. Magnet. Magnet. Mater. 1993, 128, 179. [CrossRef]

43. Schubert, M.; Tiwald, T.; Woollam, J. Explicit solutions for the optical properties of arbitrary magneto-optic materials in generalized ellipsometry. Appl. Opt. 1999, 38, 177. [CrossRef] [PubMed]

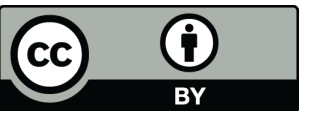

(C) 2019 by the authors. Licensee MDPI, Basel, Switzerland. This article is an open access article distributed under the terms and conditions of the Creative Commons Attribution (CC BY) license (http:/ / creativecommons.org/licenses/by/4.0/). 